\title{
Exact Finite-Size-Scaling Corrections to the Critical Two-Dimensional Ising Model on a Torus
}

\author{
Jesús Salas \\ Departamento de Física Teórica \\ Facultad de Ciencias, Universidad de Zaragoza \\ Zaragoza 50009, SPAIN \\ JESUS@MELKWEG. UNIZAR . ES
}

February 17, 2019

\begin{abstract}
We analyze the finite-size corrections to the energy and specific heat of the critical two-dimensional spin- $1 / 2$ Ising model on a torus. We extend the analysis of Ferdinand and Fisher to compute the correction of order $L^{-3}$ to the energy and the corrections of order $L^{-2}$ and $L^{-3}$ to the specific heat. We also obtain general results on the form of the finite-size corrections to these quantities: only integer powers of $L^{-1}$ occur, unmodified by logarithms; and the energy expansion contains only odd powers of $L^{-1}$. In the specific-heat expansion any power of $L^{-1}$ can appear, but the coefficients of the odd powers are proportional to the corresponding coefficients of the energy expansion.
\end{abstract}

Key Words: Ising model; finite-size scaling; corrections to scaling.

PACS Numbers: 05.50.+q, 05.70.Jk, 64.60.Cn. 


\section{Introduction}

It is well-known that phase transitions in statistical-mechanical systems can occur only in the infinite-volume limit. In any finite system, all thermodynamic quantities (such as the magnetic susceptibility and the specific heat) are analytic functions of all parameters (such as the temperature and the magnetic field); but near a critical point they display peaks whose height increases and whose width decreases as the volume $N=L^{d}$ grows, yielding the critical singularities in the limit $L \rightarrow \infty$. For bulk experimental systems (containing $N \sim 10^{23}$ particles) the finite-size rounding of the phase transition is usually beyond the experimental resolution; but in Monte Carlo simulations $\left(N \lesssim 10^{6}-10^{7}\right)$ it is visible and is often the dominant effect.

Finite-size scaling (FSS) theory [1, 2, 3, 4] provides a systematic framework for understanding finite-size effects near a critical point. The idea is simple: the only two relevant length scales are the system linear size $L$ and the correlation length $\xi_{\infty}$ of the bulk system at the same parameters, so everything is controlled by the single ratio $\xi_{\infty} / L$. If $L \gg \xi_{\infty}$, then finite-size effects are negligible; for $L \sim \xi_{\infty}$, thermodynamic singularities are rounded and obey a scaling Ansatz $\mathcal{O} \sim L^{p_{\mathcal{O}}} F_{\mathcal{O}}\left(\xi_{\infty} / L\right)$ where $p_{\mathcal{O}}$ is a critical exponent and $F_{\mathcal{O}}$ is a scaling function. Finite-size scaling is the basis of the powerful phenomenological renormalization group method (see Ref. [3] for a review); and it is an efficient tool for extrapolating finite-size data coming from Monte Carlo simulations so as to obtain accurate results on critical exponents, universal amplitude ratios and subleading exponents (see e.g. Ref. [5] for references). In particular, in systems with multiplicative and/or additive logarithmic corrections (as the twodimensional 4-state Potts model [6]), a good understanding of finite-size effects is crucial for obtaining reliable estimates of the physically interesting quantities.

In finite-size-scaling theory for systems with periodic boundary conditions, three simplifying assumptions have frequently been made:

(a) The regular part of the free energy, $f_{\text {reg }}$, is independent of the lattice size $L$ 《四 (except possibly for terms that are exponentially small in $L$ ).

(b) The scaling fields associated to the temperature and magnetic field (i.e., $g_{t}$ and $g_{h}$, respectively) are independent of $\left.L \llbracket 7\right]$.

(c) The scaling field $g_{L}$ associated to the lattice size equals $L^{-1}$ exactly, with no corrections $L^{-2}, L^{-3}, \ldots$ 叫.

Moreover, in the nearest-neighbor spin-1/2 2D Ising model, it has further been assumed that

(d) There are no irrelevant operators [8, 9].

Unfortunately, the combination of these four assumptions implies that the asymptotic expansions for the energy and specific heat for the Ising model at criticality

\footnotetext{
1 This is true only for systems below the upper critical dimension $d_{c}$. For Ising models with short-range interaction, $d_{c}=4$.
} 
terminate at order $1 / L$ (see Ref. [10]). However, the numerical results presented in 10, 5] (as well as the analytic results presented in this paper) show this to be false. The problem, therefore, is to determine which one(s) of these assumptions are invalid, and why. Assumption (c) is extremely plausible from renormalization-group considerations, at least for periodic boundary conditions; and assumption (d) has been confirmed numerically through order $\left(T-T_{c}\right)^{3}$ at least as regards the bulk behavior of the susceptibility [9]. However, both numerical [11, 12] and theoretical [13] evidence has recently emerged suggesting that irrelevant operators do contribute to the susceptibility at order $\left(T-T_{c}\right)^{4}$.

In a classic paper, Ferdinand and Fisher [14] considered the energy and the specific heat of the two-dimensional Ising model on a torus of length $L$ and aspect ratio $\rho$, and obtained the first two (resp. three) terms of the large- $L$ asymptotic expansion of the energy (resp. specific heat) at fixed $x \equiv L\left(T-T_{c}\right.$ ) [this is the finite-size-scaling regime] and fixed $\rho$. In particular, at criticality $\left(T=T_{c}\right)$ they computed the finite-size corrections to both quantities to order $L^{-1}$. In 1999, Hu et al. [15] published (without details) the correction of order $L^{-3}$ to the energy and showed that the $L^{-2}$ correction is absent.2 In this paper we compute explicitly the following finite-size corrections:

- The correction of order $L^{-3}$ to the energy.

- The corrections of order $L^{-2}$ and $L^{-3}$ to the specific heat.

Furthermore, we also find new insights into the general analytic structure of the finite-size corrections to this model. We show that in the critical two-dimensional Ising model:

- The finite-size corrections to the energy and specific heat are always integer powers of $L^{-1}$, unmodified by logarithms (except of course for the leading $\log L$ term in the specific heat).

- In the finite-size expansion of the energy, only odd integer powers of $L^{-1}$ occur.

- In the finite-size expansion of the specific heat, any integer powers of $L^{-1}$ can occur. However, the coefficients of the odd powers of $L^{-1}$ in this expansion are proportional to the corresponding coefficients in the energy expansion.

These results can be compared with the general renormalization-group expression for the finite-size corrections to the energy and the specific heat [10], in which arbitrary powers of $L^{-1}$ and terms of the type $L^{-1+p} \log L$ (where $p$ is some real number) can occur. The implications of these results for understanding which one(s) of the assumptions (a)-(d) are invalid will be analyzed elsewhere [16].

The plan of this paper is as follows: In Section 2 we present our definitions and notation (generally following Ref. 14]). In Sections 3 and 4 we present the computation of the next terms in the asymptotic expansions for the energy and specific heat,

${ }^{2}$ The published version of [15] contains several misprints in the crucial formula (3.23). Version 2 of this paper in the Los Alamos preprint archive cond-mat contains the correct formula. 
respectively. Finally, in Section 5 we present our arguments about the type of finitesize-scaling corrections that can occur in these two expansions. We have summarized in Appendix A the basic definitions and properties of the $\theta$-functions that will be needed in this paper. In Appendix B we recall the Euler-MacLaurin formula.

\section{Basic definitions}

Let us consider an Ising model on a torus of size $m \times n$ at zero magnetic field. The Hamiltonian is given by ${ }^{3}$

$$
\mathcal{H}=-K \sum_{\langle i, j\rangle} \sigma_{i} \sigma_{j}
$$

The partition function can be written as

$$
Z_{m n}=\sum_{\{\sigma\}} e^{-\mathcal{H}}=\frac{1}{2}(2 \sinh 2 K)^{m n / 2} \sum_{i=1}^{4} Z_{i}(K, n, m),
$$

where the partial partition function $Z_{1}$ is given by

$$
Z_{1}(K, n, m)=\prod_{r=0}^{n-1} 2 \cosh \left(\frac{m \gamma_{2 r+1}}{2}\right)
$$

and the rest are defined analogously using the first three columns of the following table [the last two columns will be needed afterwards in $(2.9) /(4.18)$ ].

$$
\begin{array}{ccccr}
Z_{1}: & 2 r+1 & \text { cosh } & \text { tanh } & \text { sech } \\
Z_{2}: & 2 r+1 & \text { sinh } & \text { coth } & i \text { csch } \\
Z_{3}: & 2 r & \text { cosh } & \text { tanh } & \text { sech } \\
Z_{4}: & 2 r & \text { sinh } & \text { coth } i \text { csch }
\end{array}
$$

The quantities $\gamma_{l}=\gamma_{l}(K, n)$ are defined by

$$
\cosh \gamma_{l} \equiv c_{l}=\cosh 2 K \operatorname{coth} 2 K-\cos \left(\frac{l \pi}{n}\right)
$$

In particular, we have

$$
\begin{aligned}
& \gamma_{0}=2 K+\log (\tanh K) \\
& \gamma_{l}=\log \left(c_{l}+\sqrt{c_{l}^{2}-1}\right) \quad l \neq 0
\end{aligned}
$$

The quantities $\gamma_{l}$ (2.6) satisfy $\gamma_{l}=\gamma_{2 n-l}$, and $\gamma_{l}$ is a monotonically increasing function of $l$ for $0 \leq l \leq n$.

\footnotetext{
${ }^{3}$ In this paper we are following basically the notation used by Ferdinand and Fisher in [14], with a few minor modifications.
} 
The internal energy density $E$ and the specific heat $C_{H}$ are given by

$$
\begin{aligned}
E(K, m, n) & =-\operatorname{coth} 2 K-\frac{1}{m n}\left[\frac{\sum_{i=1}^{4} Z_{i}^{\prime}}{\sum_{i=1}^{4} Z_{i}}\right] \\
C_{H}(K, m, n) & =-2 \operatorname{csch}^{2} 2 K+\frac{1}{m n}\left[\frac{\sum_{i=1}^{4} Z_{i}^{\prime \prime}}{\sum_{i=1}^{4} Z_{i}}-\left(\frac{\sum_{i=1}^{4} Z_{i}^{\prime}}{\sum_{i=1}^{4} Z_{i}}\right)^{2}\right]
\end{aligned}
$$

where the primes denote derivatives with respect to the coupling constant $K$. In computing observables (2.7)/(2.8), the following formulae, derived from (2.3), will be useful:

$$
\begin{aligned}
& \frac{Z_{1}^{\prime}}{Z_{1}}= \frac{m}{2} \sum_{r=0}^{n-1} \gamma_{2 r+1}^{\prime} \tanh \left(\frac{m \gamma_{2 r+1}}{2}\right) \\
& \frac{Z_{1}^{\prime \prime}}{Z_{1}}= {\left[\frac{m}{2} \sum_{r=0}^{n-1} \gamma_{2 r+1}^{\prime} \tanh \left(\frac{m \gamma_{2 r+1}}{2}\right)\right]^{2}+\frac{m}{2} \sum_{r=0}^{n-1} \gamma_{2 r+1}^{\prime \prime} \tanh \left(\frac{m \gamma_{2 r+1}}{2}\right) } \\
&+\left(\frac{m}{2}\right)^{2} \sum_{r=0}^{n-1}\left[\gamma_{2 r+1}^{\prime} \operatorname{sech}\left(\frac{m \gamma_{2 r+1}}{2}\right)\right]^{2}
\end{aligned}
$$

The analogous ratios for $i=2,3,4$ can be obtained from (2.9a) $/(2.9 \mathrm{~b})$ by using (2.4) [Note that the third column of (2.4) does not play any role here]. The factor $i$ in the entry $i$ csch of (2.4) changes the sign of the last term of $(2.9 \mathrm{~b})$ for $Z_{2}^{\prime \prime}$ and $Z_{4}^{\prime \prime}$.

The critical point of the Ising model corresponds to the self-dual point $\sinh 2 K_{c}=$ 1. That is

$$
K_{c}=\frac{1}{2} \log (1+\sqrt{2})
$$

In this paper we are concerned with the finite-size-scaling corrections to the energy and specific heat of the critical Ising model. We will express all our results in terms of the length $n$ and the aspect ratio of the torus $\rho:$ f $^{2}$

$$
\rho=\frac{m}{n}
$$

Indeed, all our results are invariant under the transformation $n \leftrightarrow m$. Here we shall show that the energy and specific heat at criticality have asymptotic expansions of the form

$$
\begin{aligned}
E\left(K_{c}, m, n\right) & \equiv E_{c}(n, \rho)=E_{0}+\sum_{k=1}^{\infty} \frac{E_{k}(\rho)}{n^{k}} \\
C_{H}\left(K_{c}, n, m\right) & \equiv C_{H, c}(n, \rho)=C_{00} \log n+C_{0}(\rho)+\sum_{k=1}^{\infty} \frac{C_{k}(\rho)}{n^{k}}
\end{aligned}
$$

\footnotetext{
${ }^{4}$ In conformal-field-theory language, the modular parameter of the torus is $\tau=i \rho$ [17], where this $\tau$ has nothing to do with the temperature-like parameter defined in (2.14).
} 
The coefficients $E_{0}$ and $C_{00}$ can be obtained from Onsager's solution [18]; $E_{1}, C_{0}$, and $C_{1}$ were computed by Ferdinand and Fisher [14]; and finally, the fact that $E_{2}=0$ and the expression for $E_{3}$ were given (without details) in Ref. [15]. Here we shall compute explicitly the terms $E_{3}, C_{2}$ and $C_{3}$, and shall show that $E_{2}=E_{4}=E_{6}=\ldots=0$.

Let us now see how $\gamma_{l}$ and its derivatives behave close to the critical point (2.10). To do so, we introduce the finite-size-scaling parameter $\tau$ as in Ref. [14, Eq. (2.12)]

$$
\left(\frac{\tau}{n}\right)^{2}=\frac{1}{2}\left(\sinh 2 K+\frac{1}{\sinh 2 K}\right)-1
$$

Thus, $\tau=0$ corresponds to the critical point $K=K_{c}$, and $\tau \neq 0$ fixed corresponds to the finite-size-scaling regime $n \rightarrow \infty, K \rightarrow K_{c}$ with $n\left(K-K_{c}\right)$ fixed. Hereafter, we will consider the behavior of all quantities as a function of $\tau$ in the limit $\tau \rightarrow 0$. The value of $\gamma_{0}$ at $\tau=0$ is zero; its behavior close to the critical point is given by

$$
\gamma_{0}(\tau, n)=-2\left(\frac{\tau}{n}\right)+\mathcal{O}\left[\left(\frac{\tau}{n}\right)^{3}\right]
$$

The derivatives of $\gamma_{0}$ with respect to $K$ are non-vanishing at criticality:

$$
\begin{aligned}
& \left.\gamma_{0}^{\prime}(0, n) \equiv \frac{d \gamma_{0}}{d K}\right|_{T=T_{c}}=4 \\
& \left.\gamma_{0}^{\prime \prime}(0, n) \equiv \frac{d^{2} \gamma_{0}}{d K^{2}}\right|_{T=T_{c}}=-4 \sqrt{2}
\end{aligned}
$$

(Note that prime continues to denote $d / d K$, not $d / d \tau$. However, the final result will be expressed in terms of $\tau$ [in the limit $\tau \rightarrow 0$ ], hence the notation $\left.\gamma_{0}^{\prime}(0, n)\right)$. For a generic $l \neq 0$ the critical value of $\gamma_{l}$ is given by

$$
\gamma_{l}(0, n)=2 \log \left[\sqrt{1+\sin ^{2}\left(\frac{l \pi}{2 n}\right)}+\sin \left(\frac{l \pi}{2 n}\right)\right]
$$

while its derivatives with respect to $K$ are given by

$$
\begin{aligned}
\gamma_{l}^{\prime} & =\frac{c_{l}^{\prime}}{\sqrt{c_{l}^{2}-1}} \\
\gamma_{l}^{\prime \prime} & =\frac{c_{l}^{\prime \prime}}{\sqrt{c_{l}^{2}-1}}-\frac{c_{l}\left(c_{l}^{\prime}\right)^{2}}{\left(c_{l}^{2}-1\right)^{3 / 2}}
\end{aligned}
$$

where the quantity $c_{l}^{\prime}$ vanishes at criticality as

$$
c_{l}^{\prime}(\tau, n)=c^{\prime}(\tau, n)=-8\left(\frac{\tau}{n}\right)+\mathcal{O}\left[\left(\frac{\tau}{n}\right)^{2}\right]
$$

${ }^{5}$ The parameter $\tau$ plays the same role as the usual finite-size-scaling parameter $x \equiv L\left(T-T_{c}\right)$. Indeed, to leading order in $K-K_{c}$ we have $\tau=-2 n\left(K-K_{c}\right)$. 
and the quantity $c_{l}^{\prime \prime}$ gives a non-zero value

$$
c_{l}^{\prime \prime}(0, n)=c^{\prime \prime}(0, n)=16
$$

We can write the partial partition functions $Z_{i}[$ cf. (2.3)/(2.4)] in the following form:

$$
\begin{aligned}
& Z_{1}(\tau, n, \rho)=P_{1}(\tau, n, \rho) \exp \left(\frac{m}{2} \sum_{r=0}^{n-1} \gamma_{2 r+1}\right) \\
& Z_{2}(\tau, n, \rho)=P_{2}(\tau, n, \rho) \exp \left(\frac{m}{2} \sum_{r=0}^{n-1} \gamma_{2 r+1}\right) \\
& Z_{3}(\tau, n, \rho)=P_{3}(\tau, n, \rho) \exp \left(\frac{m}{2} \sum_{r=0}^{n-1} \gamma_{2 r}\right)\left[1+e^{-m \gamma_{0}}\right] \\
& Z_{4}(\tau, n, \rho)=P_{4}(\tau, n, \rho) \exp \left(\frac{m}{2} \sum_{r=0}^{n-1} \gamma_{2 r}\right)\left[1-e^{-m \gamma_{0}}\right]
\end{aligned}
$$

where the quantities $P_{i}(\tau, n, \rho)$ are given by

$$
\begin{aligned}
& \log P_{1}(\tau, n, \rho)=\sum_{r=0}^{n-1} \log \left(1+e^{-m \gamma_{2 r+1}}\right) \\
& \log P_{2}(\tau, n, \rho)=\sum_{r=0}^{n-1} \log \left(1-e^{-m \gamma_{2 r+1}}\right) \\
& \log P_{3}(\tau, n, \rho)=\sum_{r=1}^{n-1} \log \left(1+e^{-m \gamma_{2 r}}\right) \\
& \log P_{4}(\tau, n, \rho)=\sum_{r=1}^{n-1} \log \left(1-e^{-m \gamma_{2 r}}\right)
\end{aligned}
$$

The functions (2.22) give non-vanishing constants in the limit $\tau \rightarrow 0$ [14]:

$$
\begin{aligned}
\log P_{1}(0, n, \rho) & =\frac{\theta_{3}}{\theta_{0}}+\mathcal{O}\left(n^{-2}\right) \\
\log P_{2}(0, n, \rho) & =\frac{\theta_{4}}{\theta_{0}}+\mathcal{O}\left(n^{-2}\right) \\
\log P_{3}(0, n, \rho) & =\frac{1}{2} \frac{\theta_{2}}{\theta_{0}} e^{\pi \rho / 4}+\mathcal{O}\left(n^{-2}\right) \\
\log P_{4}(0, n, \rho) & =\theta_{0}^{2}+\mathcal{O}\left(n^{-2}\right)
\end{aligned}
$$

where the functions $\theta_{i}$ with $i=2,3,4$ are the usual $\theta$-functions (see Appendix A), and $\theta_{0}$ is defined in (A.2).

Finally, we introduce the ratios

$$
R_{i}(\tau, n, \rho)=\frac{Z_{i}(\tau, n, \rho)}{Z_{1}(\tau, n, \rho)}
$$


Thus, from (2.21) we get

$$
\begin{aligned}
& R_{1}(\tau, n, \rho)=1 \\
& R_{2}(\tau, n, \rho)=\frac{P_{2}(\tau, n, \rho)}{P_{1}(\tau, n, \rho)} \\
& R_{3}(\tau, n, \rho)=2 \cosh \left(\frac{m \gamma_{0}}{2}\right) P_{0}(\tau, n, \rho) \frac{P_{3}(\tau, n, \rho)}{P_{1}(\tau, n, \rho)} \\
& R_{4}(\tau, n, \rho)=2 \sinh \left(\frac{m \gamma_{0}}{2}\right) P_{0}(\tau, n, \rho) \frac{P_{4}(\tau, n, \rho)}{P_{1}(\tau, n, \rho)}
\end{aligned}
$$

where $P_{0}(\tau, n, \rho)$ is defined as

$$
\log P_{0}(\tau, n, \rho)=\frac{m}{2}\left[\sum_{r=1}^{n-1} \gamma_{2 r}-\sum_{r=0}^{n-1} \gamma_{2 r+1}\right]
$$

The sum of the four ratios is denoted by $R$

$$
R(\tau, n, \rho)=\sum_{i=1}^{4} R_{i}(\tau, n, \rho)
$$

The ratios $R_{2}$ and $R_{3}$ have a non-vanishing value at the critical point [14]

$$
\begin{aligned}
& R_{2}(0, n, \rho)=\frac{\theta_{4}}{\theta_{3}}+\mathcal{O}\left(n^{-2}\right) \\
& R_{3}(0, n, \rho)=\frac{\theta_{2}}{\theta_{3}}+\mathcal{O}\left(n^{-2}\right)
\end{aligned}
$$

while $R_{4}$ vanishes at $K=K_{c}$ :

$$
R_{4}(\tau, n, \rho)=-\sinh (\tau \rho)\left[\theta_{2} \theta_{4}+\mathcal{O}\left(n^{-2}\right)\right]+\mathcal{O}\left(\tau^{2}\right)
$$

The sum of the four ratios at criticality is a non-zero constant

$$
R(0, n, \rho)=\frac{\theta_{2}+\theta_{3}+\theta_{4}}{\theta_{3}}+\mathcal{O}\left(n^{-2}\right)
$$

The function $P_{0}$ has also a non-vanishing limit at criticality:

$$
P_{0}(0, n, \rho)=e^{-\pi \rho / 4}\left[1+\mathcal{O}\left(n^{-2}\right)\right]
$$

\section{Finite-size-scaling corrections to the internal en- ergy}

The internal energy at the critical point $E_{c}$ is equal to

$$
-E_{c}(n, \rho)=-E\left(K_{c}, n, \rho\right)=\sqrt{2}+\lim _{\tau \rightarrow 0} \frac{1}{m n R} \sum_{i=1}^{4} \frac{Z_{i}^{\prime}}{Z_{i}} R_{i}
$$


The terms $Z_{i}^{\prime} / Z_{i}$ with $i=1,2$ vanish trivially as all the $\gamma_{2 r+1}^{\prime}$ vanish. The term $Z_{3}^{\prime} / Z_{3}$ does not vanish due to the contribution of $\gamma_{0}^{\prime}$; but its total contribution is also zero as it is multiplied by $\tanh \left(m \gamma_{0} / 2\right)$, which vanishes at criticality. The only non-vanishing contribution comes from $i=4$ :

$$
\frac{Z_{4}^{\prime}}{Z_{4}}=\frac{m}{2} \gamma_{0}^{\prime} \operatorname{coth}\left(\frac{m \gamma_{0}}{2}\right) \sim-2 m \operatorname{coth}(\rho \tau) \quad \text { as } \quad \tau \rightarrow 0
$$

So we obtain the formula

$$
-E_{c}(n, \rho)=\sqrt{2}-\frac{2}{n} \lim _{\tau \rightarrow 0} \frac{R_{4}(\tau, n, \rho)}{R(0, n, \rho)} \operatorname{coth}(\rho \tau)
$$

where $R(0, n, \rho)$ is given by (2.30). Note that, by (2.29),$R_{4}(\tau, n, \rho) \sim \tau$ as $\tau \rightarrow 0$, so $R_{4}(\tau, n, \rho) \operatorname{coth}(\rho \tau)$ gives rise to a non-zero result in this limit.

The goal of this section is to extend the Ferdinand-Fisher asymptotic expansion [14 to order $n^{-4}$. Let us first consider the quantity $\log P_{4}$ at criticality:

$$
\log P_{4}(0, n, \rho)=\sum_{r=1}^{n-1} \log \left(1-e^{-m \gamma_{2 r}}\right)=-2 \sum_{p=1}^{\infty} \frac{1}{p} \sum_{r=1}^{\left\lfloor\frac{n}{2}\right\rfloor} e^{-m p \gamma_{2 r}}
$$

where $\lfloor x\rfloor$ is the largest integer $\leq x$. The sum over $r$ can be split into two parts:

$$
\sum_{r=1}^{\left\lfloor\frac{n}{2}\right\rfloor}=\sum_{r=1}^{s(n)-1}+\sum_{r=s(n)}^{\left\lfloor\frac{n}{2}\right\rfloor}
$$

where $s(n)$ will be chosen afterwards. We can drop the second sum in (3.5), as it gives a contribution of order $\sim n\left|\log \left[1-\exp \left(-m \gamma_{2 s}\right)\right]\right| \sim n \exp [-2 \pi \rho s(n)]$. Instead of the choice $s(n)=(3 / 2 \pi \rho) \log n$ made by Ferdinand and Fisher, we shall use the choice

$$
s(n)=\frac{M}{2 \pi \rho} \log n
$$

with $M$ an arbitrary positive integer, to ensure that the total contribution of the second sum in (3.5) is as small as we want (namely, $\sim n^{-(M-1)}$ ).

We can use the following expression for $\gamma_{2 r}$ at criticality [14]:

$$
\frac{1}{2} \gamma_{2 r}(0, n)=\log \left[\sin \left(\frac{r \pi}{n}\right)+\sqrt{1+\sin ^{2}\left(\frac{r \pi}{n}\right)}\right]
$$

to obtain an asymptotic series of $m \gamma_{2 r}$ in terms of $n^{-1}$ :

$$
m \gamma_{2 r}(0, n)=2 \pi \rho r-\frac{2 \rho \pi^{3}}{3} \frac{r^{3}}{n^{2}}+\frac{\rho \pi^{5}}{3} \frac{r^{5}}{n^{4}}+\mathcal{O}\left(n^{-6}\right)
$$

We should recall that the choice of $s(n)$ (3.6) for any integer $M$ guarantees that the ratio $r / n$ is a small quantity for $0 \leq r \leq s(n)$ (as $r / n \leq s(n) / n \ll 1$ if $n$ is large enough). 
Remark: It is interesting to note that only even powers of $n^{-1}$ occur in the expansion (3.8).

Plugging the expansion (3.8) in Eq. (3.4) we obtain

$$
\log P_{4}(0, n, \rho)=-2 \sum_{p=1}^{\infty} \frac{1}{p} \sum_{r=1}^{s(n)-1} e^{-2 \pi r p \rho}-\frac{4 \pi^{3} \rho}{3} \sum_{p=1}^{\infty} \sum_{r=1}^{s(n)-1} \frac{r^{3}}{n^{2}} e^{-2 \pi r p \rho}+\mathcal{O}\left(n^{-4}\right)
$$

We can extend the sums $\sum_{r=1}^{s(n)-1}$ to $\sum_{r=1}^{\infty}$ in (3.9) by introducing an error of order $n^{-M}$. The second term of the r.h.s. of (3.9) can be expressed in terms of

$$
\sum_{r=1}^{\infty} r^{3} e^{-2 \pi r p \rho}=\frac{1}{4}\left[\frac{1}{\sinh ^{2}(\pi p \rho)}+\frac{3}{2} \frac{1}{\sinh ^{4}(\pi p \rho)}\right]
$$

Finally, we can write $P_{4}(n, \rho)$ in the following form

$$
P_{4}(0, n, \rho)=\theta_{0}^{2}\left[1-\frac{1}{n^{2}} \frac{p_{1}(\rho)}{2}+\mathcal{O}\left(n^{-4}\right)\right]
$$

which improves (2.23d). The function $p_{1}(\rho)$ is given by

$$
p_{1}(\rho)=\frac{2 \pi^{3} \rho}{3} \sum_{m=1}^{\infty}\left[\frac{1}{\sinh ^{2}(m \pi \rho)}+\frac{3}{2} \frac{1}{\sinh ^{4}(m \pi \rho)}\right]
$$

Using similar methods one can obtain the improved version of (2.23):

$$
\begin{aligned}
& P_{1}(0, n, \rho)=\frac{\theta_{3}}{\theta_{0}}\left[1-\frac{1}{n^{2}}\left(\widetilde{p}_{2}-\frac{\widetilde{p}_{1}}{2}\right)+\mathcal{O}\left(n^{-4}\right)\right] \\
& P_{2}(0, n, \rho)=\frac{\theta_{4}}{\theta_{0}}\left[1+\frac{1}{n^{2}}\left(\frac{p_{1}}{2}-p_{2}\right)+\mathcal{O}\left(n^{-4}\right)\right] \\
& P_{3}(0, n, \rho)=\frac{1}{2} \frac{\theta_{2}}{\theta_{0}} e^{\pi \rho / 4}\left[1+\frac{1}{n^{2}} \frac{\widetilde{p}_{1}}{2}+\mathcal{O}\left(n^{-4}\right)\right]
\end{aligned}
$$

where $p_{2}, \widetilde{p}_{1}$, and $\widetilde{p}_{2}$ are defined by

$$
\begin{aligned}
& \widetilde{p}_{1}(\rho)=\frac{2 \pi^{3} \rho}{3} \sum_{m=1}^{\infty}(-1)^{m+1}\left[\frac{1}{\sinh ^{2}(m \pi \rho)}+\frac{3}{2} \frac{1}{\sinh ^{4}(m \pi \rho)}\right] \\
& p_{2}(\rho)=\frac{\pi^{3} \rho}{24} \sum_{m=1}^{\infty}\left[\frac{1}{\sinh ^{2}(m \pi \rho / 2)}+\frac{3}{2} \frac{1}{\sinh ^{4}(m \pi \rho / 2)}\right] \\
& \widetilde{p}_{2}(\rho)=\frac{\pi^{3} \rho}{24} \sum_{m=1}^{\infty}(-1)^{m+1}\left[\frac{1}{\sinh ^{2}(m \pi \rho / 2)}+\frac{3}{2} \frac{1}{\sinh ^{4}(m \pi \rho / 2)}\right]
\end{aligned}
$$

Finally, we have to improve the expression of $\log P_{0}$ (2.31). Let us first consider the sum

$$
\frac{m}{2} \sum_{r=1}^{n-1} \gamma_{2 r}(0, n)=m \sum_{r=0}^{n-1} \log \left[\sin \left(\frac{r \pi}{n}\right)+\sqrt{1+\sin ^{2}\left(\frac{r \pi}{n}\right)}\right]
$$


We can apply the Euler-MacLaurin formula (B.4) to the function $f(p)=\log (\sin p+$ $\sqrt{1+\sin ^{2} p}$ ) with $L=2 n$ and $\alpha=1 / 2$. The result is

$$
\frac{m}{2} \sum_{r=1}^{n-1} \gamma_{2 r}(0, n)=\frac{2 m n}{\pi} G-\frac{\pi \rho}{6}-\frac{\pi^{3} \rho}{180} \frac{1}{n^{2}}+\mathcal{O}\left(n^{-4}\right)
$$

where $G \approx 0.915965594177219$ is Catalan's constant. Using similar methods we obtain the other sum appearing in (2.31): प

$$
\frac{m}{2} \sum_{r=0}^{n-1} \gamma_{2 r+1}(0, n)=\frac{2 m n}{\pi} G-\frac{\pi \rho}{12}-\frac{7 \pi^{3} \rho}{1440} \frac{1}{n^{2}}+\mathcal{O}\left(n^{-4}\right)
$$

Putting (3.16) and (3.17) together we obtain the improved version of (2.31)

$$
P_{0}(0, n, \rho)=e^{-\pi \rho / 4}\left[1-\frac{p_{3}(\rho)}{n^{2}}+\mathcal{O}\left(n^{-4}\right)\right]
$$

where $p_{3}(\rho)$ is defined as

$$
p_{3}(\rho)=\frac{\pi^{3}}{96} \rho
$$

The improved expressions for the ratios $R_{i}(\tau=0, n, \rho)$ are easily obtained from (3.11) / (3.13)

$$
\begin{gathered}
R_{2}(0, n, \rho)=\frac{\theta_{4}}{\theta_{3}}\left[1-\frac{1}{n^{2}}\left(p_{2}-\frac{p_{1}}{2}+\widetilde{p}_{2}-\frac{\widetilde{p}_{1}}{2}\right)+\mathcal{O}\left(n^{-4}\right)\right] \\
R_{3}(0, n, \rho)=\frac{\theta_{2}}{\theta_{3}}\left[1+\frac{1}{n^{2}}\left(\widetilde{p}_{1}-\widetilde{p}_{2}-p_{3}\right)+\mathcal{O}\left(n^{-4}\right)\right] \\
R_{4}(0, n, \rho)=-\sinh (\rho \tau) \theta_{2} \theta_{4}\left[1-\frac{1}{n^{2}}\left(\frac{p_{1}}{2}+\widetilde{p}_{2}-\frac{\widetilde{p}_{1}}{2}+p_{3}\right)\right. \\
\left.+\mathcal{O}\left(n^{-4}\right)\right]
\end{gathered}
$$

Plugging the formulae (3.20) in the expression for the critical energy density (3.1) we get

$$
-E_{c}(n, \rho)=\sqrt{2}+\frac{E_{1}(\rho)}{n}+\frac{E_{3}(\rho)}{n^{3}}+\mathcal{O}\left(\frac{1}{n^{5}}\right)
$$

where $E_{1}(\rho)$ [14] and $E_{3}(\rho)$ are given by the expressions

$$
\begin{aligned}
E_{1}(\rho)= & \frac{2 \theta_{2} \theta_{3} \theta_{4}}{\theta_{2}+\theta_{3}+\theta_{4}} \\
E_{3}(\rho)= & -\frac{2 \theta_{2} \theta_{3} \theta_{4}}{\left(\theta_{2}+\theta_{3}+\theta_{4}\right)^{2}}\left\{p_{1}(\rho)\left(\theta_{4}+\frac{\theta_{2}+\theta_{3}}{2}\right)-p_{2}(\rho) \theta_{4}\right. \\
& \left.\quad+\widetilde{p}_{1}(\rho)\left(\frac{\theta_{2}-\theta_{3}}{2}\right)+\widetilde{p}_{2}(\rho) \theta_{3}+p_{3}(\rho)\left(\theta_{3}+\theta_{4}\right)\right\}
\end{aligned}
$$

${ }^{6}$ It is easy to verify that $f^{(3)}(p)$ is integrable over $[0, \pi]$. This means that the next term in the expansion (3.16) is of order $\mathcal{O}\left(n^{-4}\right)$.

7 The leading terms of Eqs. (3.16)/(3.17) were obtained by Ferdinand 19. 
The numerical values of the function $E_{3}(\rho)$ are given in Table [

\begin{tabular}{rc}
\hline \hline$\rho$ & $E_{3}(\rho)$ \\
\hline 1 & -0.206683145336864 \\
2 & -0.184202899115749 \\
3 & -0.153247694215529 \\
4 & -0.102599506933675 \\
5 & -0.061201301359728 \\
6 & -0.034200082347112 \\
7 & -0.018369506074164 \\
8 & -0.009614465215356 \\
9 & -0.004941568941314 \\
10 & -0.002505707497764 \\
15 & -0.000074110828658 \\
20 & -0.000001946957522 \\
$\infty$ & 0 \\
\hline \hline
\end{tabular}

Table 1: Values of the coefficient $E_{3}(\rho)$ from (3.23) for several values of the torus aspect ratio $\rho$.

Remarks. 1. After the completion of this work, Prof. Izmailian informed us that the correct expression for the coefficient $E_{3}(\rho)$ had been published in the revised version of Ref. [15 (which can be found in the Los Alamos preprint archive cond-mat). Their expression is surprisingly simple

$$
E_{3}(\rho)=-\frac{\pi^{3} \rho}{48} \frac{\theta_{2} \theta_{3} \theta_{4}}{\left(\theta_{2}+\theta_{3}+\theta_{4}\right)^{2}}\left[\theta_{2}^{9}+\theta_{3}^{9}+\theta_{4}^{9}\right]
$$

Indeed, the numerical value of (3.24) coincides with our result (3.23). It would be interesting to find the analytic identities proving the equivalence of (3.23) and (3.24).

2. Let us check that (3.23) has the correct behavior under $m \leftrightarrow n(\rho \leftrightarrow 1 / \rho)$. Indeed, the (trivial) fact that $E_{c}(n, m)=E_{c}(m, n)$ implies that we should have

$$
\begin{aligned}
& E_{1}(\rho)=\frac{E_{1}(1 / \rho)}{\rho} \\
& E_{3}(\rho)=\frac{E_{3}(1 / \rho)}{\rho^{3}}
\end{aligned}
$$

The first equation (3.25a) can be easily proved by using Jacobi's imaginary transformation of the $\theta$-functions (A.4). Using these transformation one can easily show that the second equation (3.25b) holds for (3.24). We have also verified numerically that (3.25b) holds for our result (3.23) to high accuracy using MATHEMATICA.

3. There is another simple way to test our results: We can first compute the exact value of the critical energy density $E_{c}(n, \rho)$ for several values of $n$ and a fixed value of 
$\rho$ by using $(2.7) /(2.3) /(2.4)$. Then, we subtract the first two terms of the expansion (3.21) and fit the resulting function to the Ansatz $B_{3} n^{-3}+B_{5} n^{-5}+B_{7} n^{-7}$. In Table 2 we show the numerical results for such fits with $\rho=1,2$, and 3 . For $\rho=1$ we have used in the fits 1309 different values between $n=16$ and $n=4096$. For $\rho=2,3$ we have used 10 different values corresponding to $n=2^{p}, p=2, \ldots, 11$ (that is why our estimates are more accurate for $\rho=1$ than for $\rho=2,3$ ). We find an excellent agreement among the numerical estimates for $B_{3}$ and the exact values of $E_{3}$ quoted in Table 1. The value of $B_{5}$ for $\rho=1$ also agrees well with the value $\approx-0.7301823$ obtained by Izmailian [20] using analytic means.

\begin{tabular}{llll}
\hline \hline$\rho$ & \multicolumn{1}{c}{1} & \multicolumn{1}{c}{2} & \multicolumn{1}{c}{3} \\
\hline$B_{3}$ & -0.2066831453369 & -0.1842028991157 & -0.1532476942155 \\
$B_{5}$ & -0.73018231235 & -0.416996817 & -0.316738073 \\
$B_{7}$ & -4.9362 & -3.405 & -2.693 \\
\hline \hline
\end{tabular}

Table 2: Fits of the function $-E_{c}(n, \rho)-\sqrt{2}-E_{1}(\rho) / n[\mathrm{cf}$. (3.22)] to the Ansatz $B_{3} n^{-3}+B_{5} n^{-5}+B_{7} n^{-7}$ for several values of the torus aspect ratio $\rho$.

4. In the limit $\rho \rightarrow \infty$ of an infinitely long torus (i.e. a cylinder) we have

$$
E_{1}(\infty)=E_{3}(\infty)=0
$$

as $\lim _{\rho \rightarrow \infty} \rho \theta_{2}=0$, and $\lim _{\rho \rightarrow \infty} \theta_{3}=\lim _{\rho \rightarrow \infty} \theta_{4}=1$ [c.f. (A.1)].

\section{Finite-size-scaling corrections to the specific heat}

The goal of this section is to extend the asymptotic series of Ferdinand and Fisher 114 for the specific heat through order $n^{-3}$. Let us start with the definition (2.8) and see which terms contribute to the critical value of $C_{H}$. The first term in (2.8) is just a constant $(=-2)$; while the third term is quite similar to the one already obtained for the energy density (3.3) $\left[=-4 \rho R^{-2} R_{4}^{2} \operatorname{coth}^{2}(\tau \rho)\right]$. The most involved term is the second one. Using the analysis of Ferdinand and Fisher, we can get the final expression for the critical specific heat $C_{H, c}(n, \rho)=C_{H}\left(K_{c}, n, \rho\right)$ :

$$
\begin{aligned}
C_{H, c}(n, \rho)=-2+ & 4 Q_{1,-}-\frac{4 R_{3}(0, n, \rho)}{R(0, n, \rho)}\left[Q_{1,+}-Q_{1,-}\right]+4 \rho \frac{R_{3}(0, n, \rho)}{R(0, n, \rho)} \\
& -\frac{4}{R(0, n, \rho)} \sum_{i=1}^{3} R_{i}(0, n, \rho) Q_{1, i}+\frac{2 \sqrt{2}}{n} \lim _{\tau \rightarrow 0} \frac{R_{4}(\tau, n, \rho)}{R(0, n, \rho)} \operatorname{coth} \tau \rho \\
& -4 \rho \lim _{\tau \rightarrow 0}\left(\frac{R_{4}(\tau, n, \rho)}{R(0, n, \rho)} \operatorname{coth} \tau \rho\right)^{2}
\end{aligned}
$$


where the $Q_{1, \pm}$ and $Q_{1, i}$ are those defined in Ref. [14] evaluated at $\tau=0$ :

$$
\begin{aligned}
Q_{1,1}(n, \rho) & =\frac{1}{n} \sum_{r=0}^{n-1} \frac{1-\tanh \left(\frac{m \gamma_{2 r+1}}{2}\right)}{\sin \left(\frac{(r+1 / 2) \pi}{n}\right)\left[1+\sin ^{2}\left(\frac{(r+1 / 2) \pi}{n}\right)\right]^{1 / 2}} \\
Q_{1,2}(n, \rho) & =\frac{1}{n} \sum_{r=0}^{n-1} \frac{1-\operatorname{coth}\left(\frac{m \gamma_{2 r+1}}{2}\right)}{\sin \left(\frac{(r+1 / 2) \pi}{n}\right)\left[1+\sin ^{2}\left(\frac{(r+1 / 2) \pi}{n}\right)\right]^{1 / 2}} \\
Q_{1,3}(n, \rho) & =\frac{1}{n} \sum_{r=1}^{n-1} \frac{1-\tanh \left(\frac{m \gamma_{2 r}}{2}\right)}{\sin \left(\frac{r \pi}{n}\right)\left[1+\sin ^{2}\left(\frac{r \pi}{n}\right)\right]^{1 / 2}} \\
Q_{1,4}(n, \rho) & =\frac{1}{n} \sum_{r=1}^{n-1} \frac{1-\operatorname{coth}\left(\frac{m \gamma_{2 r}}{2}\right)}{\sin \left(\frac{r \pi}{n}\right)\left[1+\sin ^{2}\left(\frac{r \pi}{n}\right)\right]^{1 / 2}} \\
Q_{1,-}(n, \rho) & =\frac{1}{n} \sum_{r=0}^{n-1} \frac{1}{\sin \left(\frac{(r+1 / 2) \pi}{n}\right)\left[1+\sin ^{2}\left(\frac{(r+1 / 2) \pi}{n}\right)\right]^{1 / 2}} \\
Q_{1,+}(n, \rho) & =\frac{1}{n} \sum_{r=1}^{n-1} \frac{1}{\sin \left(\frac{r \pi}{n}\right)\left[1+\sin ^{2}\left(\frac{r \pi}{n}\right)\right]^{1 / 2}}
\end{aligned}
$$

The terms with the factors $R_{i} / R(i=3,4)$ can be obtained easily using the results of Section 3. Let us first consider the quantity $Q_{1,+}(n, \rho)$ (4.21). The first step consists in expanding the factor $\left[1+\sin ^{2}(r \pi / n)\right]^{-1 / 2}$ in Eq. (4.2f) in power series of $\sin (r \pi / n)$ :

$$
\begin{aligned}
Q_{1,+} & =\frac{1}{n} \sum_{r=1}^{n-1} \frac{1}{\sin \left(\frac{r \pi}{n}\right)}+\frac{1}{n} \sum_{r=1}^{n-1} \sum_{k=1}^{\infty}\left(\begin{array}{c}
-1 / 2 \\
k
\end{array}\right) \sin ^{2 k-1}\left(\frac{r \pi}{n}\right) \\
& \equiv Q_{1,+}^{(1)}+Q_{1,+}^{(2)}
\end{aligned}
$$

The computation of $Q_{1,+}^{(2)}$ is done by applying the Euler-MacLaurin formula $(\overline{B .4})$ to the function $f(p)=\sin ^{2 k-1}(p)$ with $L=2 n$ and $\alpha=1 / 2$. The result is

$$
Q_{1,+}^{(2)}(n, \rho)=-\frac{\log 2}{\pi}+\frac{\pi}{12} \frac{1}{n^{2}}+\mathcal{O}\left(n^{-4}\right)
$$

The computation of the divergent part $Q_{1,+}^{(1)}$ is a little more involved. The idea if to apply the Euler-MacLaurin formula (B.4) to the function $f(p)=\sin ^{-1}(p)-1 / p+$ $1 /(p-\pi)$ with $L=2 n$ and $\alpha=1 / 2$ :

$$
\begin{aligned}
\frac{1}{n} \sum_{r=0}^{n-1}\left[\sin ^{-1}\left(\frac{r \pi}{n}\right)-\frac{n}{r \pi}+\frac{n}{\pi(r-n)}\right] & =\frac{2}{\pi} \log \frac{2}{\pi}+\frac{\pi}{6 n^{2}}\left(\frac{1}{\pi^{2}}-\frac{1}{6}\right)+\mathcal{O}\left(n^{-4}\right) \\
& =Q_{1,+}^{(1)}-\frac{2}{\pi} \sum_{r=1}^{n-1} \frac{1}{r}-\frac{1}{\pi n}
\end{aligned}
$$


Using the well-known asymptotic expansion [21] [See also (5.2)]

$$
\sum_{r=1}^{L} \frac{1}{r}=\log L+\gamma_{E}+\frac{1}{2 L}-\frac{1}{12 L^{2}}+\mathcal{O}\left(L^{-4}\right)
$$

(where $\gamma_{E} \approx 0.5772156649$ is the Euler constant) we finally get

$$
Q_{1,+}^{(1)}(n, \rho)=\frac{2}{\pi}\left[\log n+\gamma_{E}+\log \frac{2}{\pi}-\frac{\pi^{2}}{72 n^{2}}+\mathcal{O}\left(n^{-4}\right)\right]
$$

Putting together (4.5)/(4.8) we arrive at the final result

$$
Q_{1,+}(n, \rho)=\frac{2}{\pi}\left[\log n+\gamma_{E}+\log \frac{2^{1 / 2}}{\pi}+\frac{\pi^{2}}{36 n^{2}}+\mathcal{O}\left(n^{-4}\right)\right]
$$

Using similar methods we obtain

$$
Q_{1,-}(n, \rho)=\frac{2}{\pi}\left[\log n+\gamma_{E}+\log \frac{2^{5 / 2}}{\pi}-\frac{\pi^{2}}{72 n^{2}}+\mathcal{O}\left(n^{-4}\right)\right]
$$

The last part consists in evaluation the $Q_{1, i}$ (with $i=1,2,3$ ) in (4.2). For brevity we will do explicitly the simplest case $Q_{1,4}(n, \rho)$ (4.2d). The first step is to expand the term $1-\operatorname{coth}\left(m \gamma_{2 r} / 2\right)$ as a power series in $\exp \left(-m \gamma_{2 r}\right)$

$$
Q_{1,4}(n, \rho)=\frac{2}{n} \sum_{r=1}^{\left\lfloor\frac{n}{2}\right\rfloor} \sum_{p=1}^{\infty} \frac{e^{-m p \gamma_{2 r}}}{\sin \left(\frac{r \pi}{n}\right) \sqrt{1+\sin ^{2}\left(\frac{r \pi}{n}\right)}}
$$

Then we split the sum over $r$ as in (3.5). The term including the sum $\sum_{r=s(n)}^{[n / 2]}$ gives a total contribution of order $n^{-(M-1)}$ with the choice (3.6) for $s(n)$. The second step is to plug into (4.11) the expansion (3.8) for $m \gamma_{2 r}$

$$
\begin{aligned}
Q_{1,4}= & -\frac{4}{n} \sum_{p=1}^{\infty} \sum_{r=1}^{s-1} \frac{e^{-2 \pi r p \rho}}{\sin \left(\frac{r \pi}{n}\right) \sqrt{1+\sin ^{2}\left(\frac{r \pi}{n}\right)}} \\
& \quad-\frac{8 \pi^{3} \rho}{3 n^{3}} \sum_{p=1}^{\infty} p \sum_{r=1}^{s-1} r^{3} \frac{e^{-2 \pi r p \rho}}{\sin \left(\frac{r \pi}{n}\right) \sqrt{1+\sin ^{2}\left(\frac{r \pi}{n}\right)}}+\mathcal{O}\left(n^{-4}\right) \\
\equiv & Q_{1,4}^{(a)}+Q_{1,4}^{(b)}
\end{aligned}
$$

The computation of $Q_{1,4}^{(b)}$ is quite easy: we expand the factors $\sin (r \pi / n)$ in powers of $r \pi / n$ :

$$
Q_{1,4}^{(b)}=-\frac{8 \pi^{2} \rho}{3 n^{2}} \sum_{p=1}^{\infty} p \sum_{r=1}^{s(n)-1} r^{2} e^{-2 \pi r p \rho}+\mathcal{O}\left(n^{-4}\right)
$$

${ }^{8}$ The leading term of this equation was obtained by Onsager [18]. 
Then we can extend the sum $\sum_{r=1}^{s(n)-1}$ to $\sum_{r=1}^{\infty}$ at an error of order $n^{-(M+2)} \log ^{2} n$. Using the fact that

$$
\sum_{p=1}^{\infty} p e^{-2 \pi r p \rho}=\frac{e^{-2 \pi r \rho}}{\left(1-e^{-2 \pi r \rho}\right)^{2}}=\sum_{p=1}^{\infty} \frac{1}{4 \sinh ^{2}(\pi r \rho)}
$$

we have that

$$
Q_{1,4}^{(b)}=-\frac{2 \pi^{2} \rho}{3 n^{2}} \sum_{p=1}^{\infty} \frac{r^{2}}{\sinh ^{2}(\pi r \rho)}
$$

The computation of $Q_{1,4}^{(a)}$ follows the same steps:

$$
Q_{1,4}^{(a)}=-\frac{4}{\pi} \sum_{p=1}^{\infty} p \sum_{r=1}^{\infty} \frac{1}{r} e^{-2 \pi r p \rho}+\frac{4 \pi}{3 n^{2}} \sum_{p=1}^{\infty} p \sum_{r=1}^{\infty} r e^{-2 \pi r p \rho}+\mathcal{O}\left(n^{-4}\right)
$$

In this case the error introduced by extending the sum $\sum_{r=1}^{s(n)-1}$ to $\sum_{r=1}^{\infty}$ is of order $n^{-M}$. Using (4.14) we find that

$$
Q_{1,4}^{(a)}=\frac{2}{\pi} \sum_{r=1}^{\infty} \frac{1}{r}[1-\operatorname{coth}(\pi r \rho)]-\frac{2 \pi}{3 n^{2}} \sum_{r=1}^{\infty} r[1-\operatorname{coth}(\pi r \rho)]+\mathcal{O}\left(n^{-4}\right)
$$

Thus, we write the final result as

$$
\begin{aligned}
Q_{1,4}(n, \rho) & =Q_{1,4}^{(0)}(\rho)+\frac{Q_{1,4}^{(2)}(\rho)}{n^{2}}+\mathcal{O}\left(n^{-4}\right) \\
Q_{1,4}^{(0)}(\rho) & =\frac{2}{\pi} \sum_{r=1}^{\infty} \frac{1}{r}[1-\operatorname{coth}(\pi r \rho)] \\
Q_{1,4}^{(2)}(\rho) & =-\frac{2 \pi}{3}\left\{\sum_{r=1}^{\infty} r[1-\operatorname{coth}(\pi r \rho)]+\pi \rho \sum_{r=1}^{\infty} r^{2}[i \operatorname{csch}(\pi r \rho)]^{2}\right\}
\end{aligned}
$$

The other three quantities $Q_{1, i}(4.2 \mathrm{a}-\mathrm{c})$ can be computed in a similar way. The result can be written as (4.18) using the translations given by (2.4) [In this case, the third column of (2.4) does not play any role]. For $i=1,2$ we should make two slight modifications: (a) The factor $r$ in (4.18) should be replaced by $r+1 / 2$, and (b) the sums over $r$ in $Q_{1,1}$ and $Q_{1,2}$ start at $r=0$ rather than at $r=1$ (as in $Q_{1,3}$ and $Q_{1,4}$ ).

Putting all the pieces together we arrive at the final result

$$
C_{H, c}(n, \rho)=\frac{8}{\pi} \log n+C_{0}(\rho)+\frac{C_{1}(\rho)}{n}+\frac{C_{2}(\rho)}{n^{2}}+\frac{C_{3}(\rho)}{n^{3}}+\mathcal{O}\left(\frac{1}{n^{4}}\right)
$$

where the coefficients $C_{i}(\rho)$ are given by

$$
C_{0}(\rho)=\frac{8}{\pi}\left(\log \frac{2^{5 / 2}}{\pi}+\gamma_{E}-\frac{\pi}{4}\right)
$$




$$
\begin{gathered}
-\frac{4}{\theta_{2}+\theta_{3}+\theta_{4}}\left[\frac{4}{\pi} \sum_{\nu=2}^{4} \theta_{\nu} \log \theta_{\nu}+\rho \frac{\theta_{2}^{2} \theta_{3}^{2} \theta_{4}^{2}}{\theta_{2}+\theta_{3}+\theta_{4}}\right] \\
C_{1}(\rho)=-2 \sqrt{2} \frac{\theta_{2} \theta_{3} \theta_{4}}{\theta_{2}+\theta_{3}+\theta_{4}}=-\sqrt{2} E_{1}(\rho) \\
C_{2}(\rho)=-4 \rho \frac{\theta_{2} \theta_{3} \theta_{4}}{\theta_{2}+\theta_{3}+\theta_{4}} E_{3}(\rho)-\frac{\pi}{9}-4 A_{3}(\rho) \frac{\theta_{2}}{\theta_{2}+\theta_{3}+\theta_{4}}\left(\rho-\frac{\log 16}{\pi}\right) \\
+\frac{\pi}{3} \frac{\theta_{2}}{\theta_{2}+\theta_{3}+\theta_{4}}-\frac{16}{\pi}\left[A_{2}(\rho) \theta_{4}+A_{3}(\rho) \theta_{2}\right] \frac{\nu=2}{\left(\theta_{2}+\theta_{3}+\theta_{4}\right)^{2}} \\
-\frac{4}{\theta_{2}+\theta_{3}+\theta_{4}} G(\rho) \\
C_{3}(\rho)=-\sqrt{2} E_{3}(\rho)
\end{gathered}
$$

where

$$
\begin{aligned}
G(\rho) & =Q_{1,1}^{(2)} \theta_{3}+Q_{1,2}^{(2)} \theta_{4}+Q_{1,3}^{(2)} \theta_{2}-Q_{1,2}^{(0)} A_{2}(\rho) \theta_{4}-Q_{1,3}^{(0)} A_{3}(\rho) \theta_{2} \\
A_{2}(\rho) & =p_{2}(\rho)+\widetilde{p}_{2}(\rho)-\frac{1}{2}\left[p_{1}(\rho)+\widetilde{p}_{1}(\rho)\right] \\
A_{3}(\rho) & =p_{3}(\rho)+\widetilde{p}_{2}(\rho)-p_{1}(\rho)
\end{aligned}
$$

The expressions for $C_{0}$ and $C_{1}$ were first obtained by Ferdinand and Fisher [14]. The results for $C_{2}$ and $C_{3}$ are new. In Table 3 we show the values of the coefficient $C_{2}(\rho)$ for several values of the aspect ratio $\rho$.

\begin{tabular}{rr}
\hline \hline$\rho$ & $C_{2}(\rho)$ \\
\hline 1 & 0.097119896855337 \\
2 & -0.326865280829340 \\
3 & -0.748187561687100 \\
4 & -0.877385104391125 \\
5 & -0.809168407448959 \\
6 & -0.682469414146146 \\
7 & -0.567445479079586 \\
8 & -0.483401539198706 \\
9 & -0.428249598449714 \\
10 & -0.394311952593824 \\
15 & -0.351150319692501 \\
20 & -0.349140134316672 \\
$\infty$ & -0.349065850398866 \\
\hline \hline
\end{tabular}

Table 3: Values of the coefficient $C_{2}(\rho)$ from (4.22) for several values of the torus aspect ratio $\rho$.

Remarks 1. As shown by Ferdinand and Fisher using the Jacobi's transformations 
(A.4), the coefficient $C_{0}(\rho)$ satisfies the identity

$$
C_{0}(\rho)=C_{0}\left(\rho^{-1}\right)+\frac{8}{\pi} \log \rho
$$

Indeed, from relations (4.21)/(4.23) we conclude that these two coefficients have the right behavior under the transformation $\rho \rightarrow 1 / \rho$ [c.f.,(3.25a) $/(3.25 b)]$. Finally, we have tested numerically that

$$
C_{2}(\rho)=\frac{C_{2}(1 / \rho)}{\rho^{2}}
$$

is satisfied. This is a non-trivial test of the correctness of our result.

2. We have also performed the following test: we have defined the function equal to the exact value of the specific heat $C_{H, c}(n, \rho)$ minus the first three terms of the expansion (4.19). Then, we have fitted the result to several Ansätze. In particular, we show in Table 1 the results for the Anstaz $D_{2} / n^{2}+D_{3} / n^{3}+D_{4} / n^{4}+D_{5} / n^{5}$. We have used the same data as for the enrgy fits in Section 3. The agreement between the values $D_{2}, D_{3}$ and the exact values $(4.22) /(4.23) /(3.23)$ is very good. It is also interesting to note that $D_{5}(\rho) \approx-\sqrt{2} B_{5}(\rho)$, where $B_{5}(\rho)$ is the coefficient obtained in a similar fit to the energy (see Table 2).

\begin{tabular}{llll}
\hline \hline$\rho$ & \multicolumn{1}{c}{1} & \multicolumn{1}{c}{2} & \multicolumn{1}{c}{3} \\
\hline$D_{2}$ & 0.097119896855 & -0.3268652808 & -0.7481875617 \\
$D_{3}$ & 0.2922941107 & 0.2605022 & 0.2167250 \\
$D_{4}$ & 0.014792 & -1.0635 & -1.8668 \\
$D_{5}$ & 1.0326 & 0.59 & 0.45 \\
\hline \hline
\end{tabular}

Table 4: Fits of the function $C_{H, c}(n, \rho)-(8 / \pi) \log n-C_{0}(\rho)-C_{1}(\rho) / n[\mathrm{cf}$. $(4.19) /(4.20) /(4.21)]$ to the Ansatz $D_{2} n^{-2}+D_{3} n^{-3}+D_{4} n^{-4}+D_{5} n^{-5}$ for several values of the torus aspect ratio $\rho$.

3. By inspection from Table [3, the function $C_{2}(\rho)$ should have a zero at a nontrivial value of the aspect ratio $\rho_{\text {min }}$ between 1 and 2 . We have evaluated numerically that value

$$
\rho_{\text {min }} \approx 1.33544086
$$

By (4.26), there is another zero of $C_{2}(\rho)$ at $\rho_{\min }^{-1} \approx 0.74881639$.

4. In the limit $\rho \rightarrow \infty$ the coefficients $C_{i}$ tend to the following limits:

$$
\begin{aligned}
& C_{0}(\infty)=\frac{8}{\pi}\left(\log \frac{2^{5 / 2}}{\pi}+\gamma_{E}-\frac{\pi}{4}\right) \\
& C_{1}(\infty)=0 \\
& C_{2}(\infty)=-\frac{\pi}{9} \\
& C_{3}(\infty)=0
\end{aligned}
$$

Thus, only the coefficients associated to even powers of $n^{-1}$ survive in this limit. 


\section{$5 \quad$ Further remarks and conclusions}

The computation of the finite-size corrections to the energy and specific heat shows that, through order $n^{-3}$ :

(a) All the corrections are integer powers of the quantity $n^{-1}$. In particular there are no multiplicative or additive logarithmic terms (except for the leading term in the specific heat).

(b) In the energy density we only find odd powers of $n^{-1}$. In particular, the corrections of order $n^{-2}$ and $n^{-4}$ are absent in (3.21).

(c) In the specific heat we find both even and odd powers of $n^{-1}$. But the coefficients of the corrections corresponding to odd powers of $n^{-1}$ in (4.19) are proportional to the corresponding coefficients in the energy expansion (3.21): we found that $C_{i}(\rho)=-\sqrt{2} E_{i}(\rho)$ for $i=1,3[\mathrm{cf}$. (4.21)/(4.23)], and the numerical test performed at the end of Sections 3 and 1 shows that the same ratio holds for the next coefficients $C_{5} / E_{5} \approx-\sqrt{2}$.

The natural question is whether these observations are general features that hold to all orders in $n^{-1}$. In this section, we will try to answer those questions.

Let us first analyze what happens to the energy. We first note that in the expansion (3.8) only even powers of $n^{-1}$ occur. This expansion can be done to any finite order we want. The errors coming from neglecting the second sum in (3.5) and from extending the sums $\sum_{r=1}^{s(n)-1}$ to $\sum_{r=1}^{\infty}$ are at most of order $\mathcal{O}\left(n^{-(M-1)}\right)$, and they can be made as small as we want by making $M$ in the definition of $s(n)$ (3.6) as large as we need. This means that in the series expansions of the quantities $\log P_{i}(i=1, \ldots, 4)(3.13)$ only even powers of $n^{-1}$ appear. Secondly, we need to check that only even powers of $n^{-1}$ occur in the expansion of $\log P_{0}(3.15)$. The argument is simple: all derivatives $f^{(k)}$ of the function $f(p)=\log \left(\sin p+\sqrt{1+\sin ^{2} p}\right)$ are integrable over the interval $[0, \pi]$. This implies that the Euler-MacLaurin formula (B.4) can be applied to any arbitrary order. As $f(0)=f(\pi)$, the correction of order $n^{-1}$ vanishes and only corrections with even powers of $n^{-1}$ can occur. The same conclusion applies to the expansion (3.15) and to the ratios $R_{i}(3.20)$. Thus, from formula (3.3) we immediately conclude that only odd powers of $n^{-1}$ appear in the finite-size corrections to the critical energy density. This result generalizes point (b) above. In particular, no logarithmic corrections occur in this expansion at any order.

The analysis of the specific heat is a little more involved. Using the same procedure as for the energy, we conclude that the fourth $\left(\sim R_{3} / R\right)$ and the last $\left(\sim R_{4}^{2} / R^{2}\right)$ terms of (4.1) provide corrections with even powers of $n^{-1}$. Furthermore, the sixth term of (4.1] $\left[\sim R_{4} /(R n)\right]$ will give only odd powers of $n^{-1}$.

Let us see nor what happens to the terms $Q_{1, i}(4.2 \mathrm{a}-\mathrm{d})$. The argument for $Q_{1,4}$ (4.2d) is quite simple: the expansion of this quantity as a power series of $r \pi / n$ will only contain even powers of $n^{-1}$. Indeed, the errors coming from dropping the sum $\sum_{r=s(n)}^{\lfloor n / 2\rfloor}$ in the beginning of the computation, and for extending the sum $\sum_{r=1}^{s(n)-1}$ to $\sum_{r=1}^{\infty}$ at the end of the computation are both at most of order $\mathcal{O}\left(x^{-(M-1)}\right)$ with the 
choice (3.6) for $s(n)$. Thus, the fifth term in (4.1) $\left[\sim \sum R_{i} Q_{1, i} / R\right]$ will have only corrections with even powers of $n^{-1}$.

Finally, let us analyze the behavior of $Q_{1,+}(4.21)$. In the evaluation of $Q_{1,+}^{(2)}$ we had to apply the Euler-MacLaurin formula (B.4) to the function $f(p)=\sin ^{2 k-1} p$ with $k \geq 1$. The derivatives of this function are always integrable over the interval $[0, \pi]$, thus the expansion (4.5) only contains even powers of $n^{-1}$. In the evaluation of $Q_{1,+}^{(0)}$ we apply the Euler-MacLaurin formula (B.4) to the function $f(p)=1 / \sin p-1 / p+$ $1 /(p-\pi)$. This function and all its derivatives are integrable over the interval $[0, \pi]$. Thus, Eq. (4.6) can be generalized to

$$
Q_{1,+}^{(0)}=\frac{2}{\pi}\left\{\sum_{p=1}^{n-1} \frac{1}{p}+\frac{1}{2 n}+\log \frac{2}{\pi}+\frac{\pi}{2} \sum_{k=1}^{\infty} \frac{B_{2 k}}{2 k}\left(\frac{\pi}{n}\right)^{2 k}\left[f^{(2 k-1)}(\pi)-f^{(2 k-1)}(0)\right]\right\}
$$

This expansion contains in principle both even and odd powers of $n^{-1}$. We now plug in the well-known result [21]

$$
\sum_{p=1}^{L} \frac{1}{p}=\log L+\gamma_{E}+\frac{1}{2 L}-\sum_{k=1}^{\infty} \frac{B_{2 k}}{2 k} L^{-2 k}
$$

with $L=n-1$, and expand the resulting factors $\log (1-1 / n),(1-1 / n)^{-1}$, and $(1-1 / n)^{-2 k}$ in powers of $n^{-1}$. If we express the result as $\sum_{k} \alpha_{k} n^{-k}$, we immediately see that $\alpha_{1}=0$. The expression for the coefficients of the odd powers of $n^{-1}$ is given by

$$
\alpha_{2 k+1}=\frac{1}{2}-\frac{1}{2 k+1}-\sum_{m=1}^{k}\left(\begin{array}{c}
2 k \\
2 m-1
\end{array}\right) \frac{B_{2 m}}{2 m}, \quad k \geq 1
$$

To prove $\alpha_{2 k+1}=0$, we can apply the general Euler-MacLaurin formula (B.1) to the function $f(x)=x^{2 k}$ with $n=0$ and $m=1$ :

$$
0=\int_{0}^{1} x^{2 k} d x-\frac{1}{2}+\sum_{m=1}^{k} \frac{B_{2 m}}{(2 m) !} \frac{(2 k) !}{(2 k-2 m+1) !}=-\alpha_{2 k+1}
$$

This implies that in the finite-size-scaling expansion of $Q_{1,+}$ only even powers of $n^{-1}$ occur. The same holds for $Q_{1,-}$ (4.2e).

In summary, we have seen that in the finite-size-scaling expansion of the specific heat at criticality only integer powers of $n^{-1}$ appear [except of course for the leading term $(8 / \pi) \log n]$. Furthermore, all contributions to the specific heat (4.1) give even powers of $n^{-1}$, except for one: namely, the sixth term in (4.1), which has the form

$$
\frac{2 \sqrt{2}}{n} \lim _{\tau \rightarrow 0} \frac{R_{4}(\tau, n, \rho)}{R(0, n, \rho)} \operatorname{coth} \tau \rho
$$

If we compare it to the expression for the energy (3.3) we conclude that the coefficients associated to odd powers of $n^{-1}$ in the energy and specific-heat expansions are proportional. In particular [c.f., (2.12)/(2.13)],

$$
\frac{C_{2 k+1}}{E_{2 k+1}}=-\sqrt{2} \quad \forall k \geq 0
$$

This generalizes the results (4.21)/(4.23). 


\section{A Theta functions}

In this appendix we gather all the definitions and properties of the Jacobi's $\theta$ functions needed in this paper. We follow the notation of Ref. [14], which was adapted from Whittaker and Watson [22]. We define the Jacobi $\theta$-functions $\theta_{i}$ at $z=0$ in the following way:

$$
\begin{aligned}
& \theta_{2} \equiv \theta_{2}\left(0, e^{-\pi \rho}\right)=2 \theta_{0} e^{-\pi \rho / 4} \prod_{r=1}^{\infty}\left(1+e^{-2 r \pi \rho}\right)^{2} \\
& \theta_{3} \equiv \theta_{3}\left(0, e^{-\pi \rho}\right)=\theta_{0} \prod_{r=1}^{\infty}\left(1+e^{-(2 r-1) \pi \rho}\right)^{2} \\
& \theta_{4} \equiv \theta_{4}\left(0, e^{-\pi \rho}\right)=\theta_{0} \prod_{r=1}^{\infty}\left(1-e^{-(2 r-1) \pi \rho}\right)^{2}
\end{aligned}
$$

The function $\theta_{0}(\rho)$ is defined as

$$
\theta_{0}=\theta_{0}(\rho)=\prod_{r=1}^{\infty}\left(1-e^{-2 \pi r \rho}\right)
$$

and it satisfies the following identity

$$
\theta_{0}=e^{\pi \rho / 12}\left[\frac{1}{2} \theta_{2} \theta_{3} \theta_{4}\right]^{1 / 3}
$$

These $\theta$-functions (A.1) satisfy the Jacobi's imaginary transformation

$$
\begin{aligned}
& \theta_{2}\left(0, e^{-\pi / \rho}\right)=\rho^{1 / 2} \theta_{4}\left(0, e^{-\pi \rho}\right) \\
& \theta_{3}\left(0, e^{-\pi / \rho}\right)=\rho^{1 / 2} \theta_{3}\left(0, e^{-\pi \rho}\right) \\
& \theta_{4}\left(0, e^{-\pi / \rho}\right)=\rho^{1 / 2} \theta_{2}\left(0, e^{-\pi \rho}\right)
\end{aligned}
$$

\section{B The Euler-MacLaurin formula}

The Euler-MacLaurin formula (see e.g. [23, Appendix B]) is the main tool we need to compute asymptotic series. The general form of this formula is given by

$$
\begin{array}{rl}
\sum_{k=n}^{m-1} f(k)=\int_{n}^{m} & d x f(x)-\frac{1}{2}[f(m)-f(n)] \\
& +\sum_{p=1}^{N} \frac{B_{2 p}}{(2 p) !}\left[f^{(2 p-1)}(m)-f^{(2 p-1)}(n)\right] \\
& +\frac{1}{(2 N+1) !} \int_{n}^{m} d x f^{(2 N+1)}(x) B_{2 N+1}(x-\lfloor x\rfloor)
\end{array}
$$

${ }^{9}$ In this particular case, $\theta_{1}\left(0, e^{-\pi \rho}\right)=0$. 
where $B_{n}$ are the Bernoulli numbers and $B_{n}(x)$ are the Bernoulli polynomials defined by

$$
B_{n}(x)=\sum_{k=0}^{n}\left(\begin{array}{l}
n \\
k
\end{array}\right) B_{k} x^{n-k}
$$

In this paper we are mainly interested in sums of the form

$$
\frac{1}{L} \sum_{n=0}^{\alpha L-1} f(p)
$$

where $p=2 \pi n / L$. The asymptotic expansion of the sum (B.3) in the limit $L \rightarrow \infty$ with $\alpha$ fixed can be obtained from (B.1):

$$
\begin{aligned}
\frac{1}{L} \sum_{n=0}^{\alpha L-1} f(p)=\int_{0}^{2 \pi \alpha} & \frac{d p}{2 \pi} f(p)-\frac{1}{2 L}[f(2 \pi \alpha)-f(0)] \\
& +\frac{1}{2 \pi} \sum_{k=1}^{N} \frac{B_{2 k}}{(2 k) !}\left(\frac{2 \pi}{L}\right)^{2 k}\left[f^{(2 k-1)}(2 \pi \alpha)-f^{(2 k-1)}(0)\right] \\
& +\frac{1}{(2 N+1) !}\left(\frac{2 \pi}{L}\right)^{2 N+1} \int_{0}^{2 \pi \alpha} \frac{d p}{2 \pi} f^{(2 N+1)}(p) \widehat{B}_{2 N+1}(p)
\end{aligned}
$$

where

$$
\widehat{B}_{n}(p)=B_{n}\left(\frac{L p}{2 \pi}-\left\lfloor\frac{L p}{2 \pi}\right\rfloor\right)
$$

The expression (B.4) gives the asymptotic expansion of the sum (B.3) in powers of $L^{-1}$ up to order $L^{-2 N}$ if the last integral in (B.4) is finite (i.e., if $f^{(2 N+1)}(p)$ is integrable in the interval $[0,2 \alpha \pi])$. If $f(0)=f(2 \pi \alpha)$, then only even powers of $L^{-1}$ occur in the expansion (B.4).

\section{Acknowledgments}

We wish to thank Nickolay Izmailian for correspondence and useful clarifications about Ref. [15]; Robert Shrock for his warm hospitality and discussions during the authors' visit to the C.N. Yang Institute for Theoretical Physics, where this work was mainly done; and Alan Sokal for useful discussions and for a critical reading of the first draft of this manuscript. The authors' research was supported in part by CICyT (Spain) grants AEN97-1680 and AEN99-0990.

\section{References}

[1] M.E. Fisher, in Critical Phenomena, Proc. 51th Enrico Fermi Summer School, Varena, edited by M.S. Green (Acedemic Press, New York, 1972).

[2] M.E. Fisher and M.N. Barber, Phys. Rev. Lett. 28 (1972) 1516. 
[3] M.N. Barber, in Phase Transitions and Critical Phenomena, Vol. 8, edited by C. Domb and J.L. Lebowitz (Academic Press, New York, 1983).

[4] V. Privman in Finite Size Scaling and Numerical Simulation of Statistical Systems, edited by V. Privman (World Scientific, Singapore, 1990).

[5] J. Salas and A.D. Sokal, J. Stat. Phys. 98 (2000) 551, cond-mat/9904038r2.

[6] J. Salas and A.D. Sokal, J. Stat. Phys. 88, 567 (1997), hep-lat/9607030.

[7] H. Guo and D. Jasnow, Phys. Rev. B 35, 1846 (1987); 39, 753 (E) (1989).

[8] A. Aharony and M.E. Fisher, Phys. Rev. B 27, 4394 (1983).

[9] S. Gartenhaus and W.S. McCullough, Phys. Rev. B 38, 11688 (1988).

[10] J. Salas and A.D. Sokal, Universal amplitude ratios in the critical twodimensional Ising model on a torus, cond-mat/9904038v1.

[11] B. Nickel, J. Phys. A 32 (1999) 3889.

[12] B. Nickel, J. Phys. A 33 (2000) 1693.

[13] M. Caselle, M. Hasenbusch, A. Pelissetto and E. Vicari, High-precision estimate of $g_{4}$ in the 2D Ising model, hep-th/0003049.

[14] A.E. Ferdinand and M.E. Fisher, Phys. Rev. 185, 832 (1969).

[15] C.-K. Hu, J.-A. Chen, N. Sh. Izmailian and P. Kleban, Phys. Rev. E 60 (1999) 6491, cond-mat/9905203v2.

[16] J. Salas and A.D. Sokal, in preparation.

[17] C. Itzykson and J.-M. Drouffe, Statistical Field Theory (Cambridge University Press, Cambridge, 1989), Vol. 2.

[18] L. Onsager, Phys. Rev. 65, 117 (1944).

[19] A.E. Ferdinand, J. Math. Phys. 8 (1967) 2332.

[20] N. Sh. Izmailian, private communication (August 2000).

[21] I.S. Gradshteyn and I.M. Ryzhik, Table of Integrals, Series and Products (Academic Press, New York, 1965).

[22] E.T. Whittaker and G.N. Watson, A Course of Modern Analysis, 4th ed. (Cambridge University Press, Cambridge, 1927).

[23] S. Caracciolo and A. Pelissetto, Phys. Rev. D 58 (1998) 105007. 